\title{
Effect of polyaniline and aluminum tri-polyphosphate particles addition on the protective properties of Mg-rich epoxy coating on AZ91D magnesium alloy
}

\author{
Xiangyu Lu ${ }^{l \#}$, Xiao Wang ${ }^{2 \#}$, Song Chen ${ }^{1}$, Jiatao Cai ${ }^{1}$, Hong Tao ${ }^{1}$, Zhikai Xu ${ }^{1}$, Xingguo Feng ${ }^{1 *}$ \\ ${ }^{1}$ Key Laboratory of Coastal Disaster and Defence (Hohai University), Ministry of Education, Nanjing \\ 210024 Jiangsu, China \\ ${ }^{2}$ Marine Chemical Research Institute, State Key Laboratory of Marine Coatings, Qingdao, 266072, \\ China \\ \# Xiangyu Lu and Xiao Wang are co-first authors of the article. \\ *E-mail: fengxingguo@hhu.edu.cn
}

Received: 6 September 2021 / Accepted: 8 October 2021 / Published: 10 November 2021

\begin{abstract}
Hydrofluoric acid doped PANI (PANI-HF) and aluminum tri-polyphosphate (SAP) particles were together added into a Mg-rich primer (MRP) on the AZ91D magnesium alloy and improved the protective performance of the MRP greatly. The coating performance was studied with Machu test, OCP and EIS. The surface analysis was conducted on the coating and the AZ91D substrate using SEM and XPS. The results showed that the protective effect of the PANI-HF/SAP/MRP was better than that of the MRP for the substrate underneath the defect coating. On one hand, the corrosion products, consisting of magnesium oxides and phosphates, precipitated on the Mg particles, which healed the micro defects in the coating and improved its barrier effects. On the other hand, a protective film was formed on the AZ91D substrate, which could prevent the permeation of the electrolyte and retard the corrosion of the substrate.
\end{abstract}

Keywords: Magnesium alloy; Mg-rich epoxy coating; Polyaniline; Aluminum Tri-polyphosphate; EIS

\section{$\underline{\text { FULL TEXT }}$}

(C) 2021 The Authors. Published by ESG (www.electrochemsci.org). This article is an open access article distributed under the terms and conditions of the Creative Commons Attribution license (http://creativecommons.org/licenses/by/4.0/). 